\title{
Síntesis de calostro y desarrollo de las crías por efecto de la inclusión de aceite de soya en la dieta preparto de ovejas de pelo
}

\section{Colostrum synthesis and development of lambs by effect of soybean oil inclusion in the pre-partum diet of hair ewes}

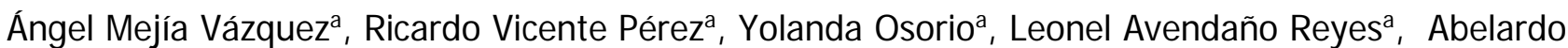 \\ Correa Calderóna, Francisco D. Álvareza , Ulises Macías Cruzª
}

\begin{abstract}
RESUMEN
El objetivo fue evaluar el efecto del nivel de inclusión de aceite de soya (AS) en la dieta pre-parto de ovejas de pelo sobre la producción y composición del calostro, desarrollo de las crías en los primeros 10 días postparto y la productividad de la oveja al parto. Un grupo de ovejas multíparas preñadas Katahdin x Pelibuey se asignaron a tres tratamientos ( $n=10)$, los cuales consistieron en dietas isoenergéticas e isoprotéicas que contenían: 0,3 y $6 \%$ de AS/ $\mathrm{kg}$ de materia seca. Las dietas se ofrecieron del día 100 de gestación al parto. El peso y volumen del calostro aumentaron linealmente $(P<0.05)$ conforme el nivel de AS en la dieta incrementó. El resto de las variables no presentaron efectos lineales ni cuadráticos $(P>0.05)$ por nivel de AS. Entre ovejas alimentadas con y sin AS, o entre ovejas alimentadas con 3 y $6 \%$ de AS, no se observaron diferencias ( $P>0.05$ ) en peso vivo y condición corporal de las ovejas, así como en la composición del calostro, peso al nacimiento de las crías y el peso de camada al parto. Se concluye que la adición de $306 \%$ de AS en la dieta preparto de ovejas de pelo no afectó la composición del calostro, el desarrollo de las crías ni la productividad de la oveja, pero sí afectó la producción de calostro, la cual fue mayor al nivel de $6 \%$ de AS en la dieta.
\end{abstract}

PALABRAS CLAVE: Ovejas preñadas, Nutrición materna, Aceite de soya, Producción de calostro.

\begin{abstract}
The objective was to evaluate the effect of the inclusion level of soybean oil (SBO) in the prepartum diet of hair ewes on the colostrum production and composition, development of lambs at $\mathbf{d} \mathbf{1 0}$ post-lambing and ewe productivity at lambing. A group of pregnant multiparous ewes from Katahdin x Pelibuey genotype were assigned to three treatments ( $n=10)$, which consisted of isoenergetic and isoproteic diets containing: 0, 3 and $6 \%$ of SBO/ $\mathrm{kg}$ dry matter. Diets were offered from d 100 of gestation to lambing. The colostrum weight and volume increased linearly $(P<0.05)$ as the SBO level in diet increased. The rest of variables did not show any linear or quadratic effect $(P>0.05)$ as the SBO level changed from 0 to $6 \%$. Between ewes fed with or without SBO, or between ewes fed 3 and $6 \%$ of SBO, no difference was detected $(P>0.05)$ on live weight and body condition score of ewes, as well as on colostrum composition, lamb birth weight and litter weight at lambing. In conclusion, the addition of 3 or $6 \%$ of SBO to prepartum diets of hair ewes did not affect the colostrum composition, the development of lambs or the ewe productivity, but affected the colostrum production, being greater when $6 \%$ of SBO was used in the diet.
\end{abstract}

KEY WORDS: Pregnant ewes, Maternal nutrition, Soybean oil, Calostrum production.

Recibido el 17 de noviembre de 2015. Aceptado el 14 de marzo de 2016.

a Instituto de Ciencias Agrícolas, Universidad Autónoma de Baja California, Valle de Mexicali, B.C., 21705, México.

*Autor de correspondencia ulisesmacias1988@hotmail.com.

Apoyo recibido: Investigación financiada en la 17va. Convocatoria Interna de Apoyo a Proyectos de Investigación UABC. 


\section{INTRODUCCIÓN}

La productividad de los rebaños en México es mermada por la muerte postnatal de corderos, principalmente en las primeras horas o días postparto. Algunos estudios sugieren que varios de los factores predisponentes de la muerte prenatal de corderos (peso al nacimiento, síntesis de calostro y vigor del cordero, entre otros), podrían ser controlados a través de una alimentación adecuada en el último tercio de gestación ${ }^{(1,2)}$, específicamente usando aceites vegetales como ingrediente en la dieta preparto de las ovejas ${ }^{(3)}$.

Los aceites vegetales son ricos en ácidos grasos polinsaturados de cadena larga, los cuales son esenciales en el desarrollo del sistema nervioso e inmunológico de fetos en crecimiento de las especies mamíferas ${ }^{(4,5)}$. Así, cuando las ovejas gestantes en el último tercio se alimentan con dietas ricas en ácidos grasos polinsaturados, favorecen el vigor al nacimiento ${ }^{(6)}$ y disminuyen la tasa de mortalidad postnatal en los corderos ${ }^{(5)}$. Adicionalmente, en las ovejas mejora el estado oxidativo(7) y la respuesta inmune ${ }^{(8)}$. Sin embargo, niveles excesivos de aceites vegetales en la dieta pueden conducir a efectos negativos sobre el trabajo de parto, el peso al nacimiento de las crías, la composición del calostro y la ganancia de peso pre- y postparto de la oveja.

En ovejas, la síntesis de prostaglandinas $E_{2}$ y $F_{2 a}$ placentarias se incrementa por la adición de aceites vegetales en la dieta preparto, situación que favorece la incidencia de partos prematuros ${ }^{(9)}$. Los corderos que nacen prematuramente se caracterizan por ser débiles y con bajos pesos al nacimiento ${ }^{(10)}$. También, se ha documentado que la inclusión de altos niveles de aceites vegetales en la dieta de rumiantes tiene efectos negativos sobre la población microbiana y la digestibilidad de los nutrientes por una excesiva biohidrogenación ruminal(11), lo cual puede comprometer la disponibilidad de nutrientes para el crecimiento fetal. Asimismo, algunos estudios $^{(12,13)}$ señalan que en rumiantes lactando, la producción de leche y el contenido de grasa en leche se reducen con un nivel elevado de aceites vegetales dietarios, ya que se favorece un incremento en la síntesis del ácido graso $\mathrm{C} 18: 2$ trans-10 cis-12 durante la biohidrogenación ruminal(14). Así, es posible que se puedan encontrar efectos similares sobre la síntesis de calostro en ovejas u otros rumiantes alimentados con un nivel inadecuado de aceite vegetal, aunque no se encontraron evidencias al respecto.

Cabe mencionar que poco se ha investigado en relación al impacto que tiene el uso de aceites vegetales en la dieta preparto sobre la síntesis de calostro, el peso al nacimiento de las crías y el desarrollo de los corderos en los primeros días postparto en ovejas de razas de pelo. Por lo tanto, el objetivo del estudio fue evaluar el efecto del nivel de inclusión de aceite de soya (AS) en la dieta preparto de ovejas de pelo sobre la síntesis de calostro, el desarrollo de las crías en los primeros días postparto y la productividad de la oveja al parto.

\section{MATERIAL Y MÉTODOS}

El experimento se realizó durante la época de primavera en la Unidad Experimental Ovina del Instituto de Ciencias Agrícolas-UABC, ubicada en el Valle de Mexicali, Baja California, México. El clima en la región se clasifica como árido, muy caluroso y seco (BWh).

\section{Manejo pre-experimental}

Un grupo de 40 ovejas multíparas Katahdin $\mathrm{x}$ Pelibuey se empadró usando la metodología descrita por Vicente-Pérez et $a^{\left({ }^{2}\right)}$, la cual considera la aplicación de un protocolo de sincronización de estro en combinación con un sistema de montas naturales controladas. Esto garantizó que todas las ovejas diagnosticadas gestantes $(95 \%)$ al día 50 postmonta presentaran un tiempo de gestación de 100 días al inicio del experimento. El diagnóstico de preñez se realizó con la ayuda de un ultrasonido portátil (Draminski, modelo Animal Profi, transductor rectal multifrecuencial de 3.5 a $7.5 \mathrm{Mhz}$ ). Antes de iniciar el protocolo de sincronización (10 días), todas las ovejas se trataron individualmente con desparasitante (Ivermectina, Sanfer ${ }^{\circledR}$ ) y vitaminas (Vigantol ADE, Bayer ${ }^{\circledR}$ ). En general, las ovejas en el periodo pre-experimental (día 30 al 99 post-servicio) se mantuvieron estabuladas en dos corrales y se alimentaron con paja de trigo picada ad libitum más $300 \mathrm{~g} /$ día de un concentrado formulado con $48.5 \%$ 
de trigo molido, $48.5 \%$ de pasta de soya y $3.0 \%$ de premezcla de vitaminas-minerales. La disponibilidad del agua fue a libre acceso. Antes de iniciar el experimento (entre el día 90 y 99 de gestación), todas las ovejas se adaptaron al consumo de AS, incrementando en forma paulatina el nivel de aceite hasta llegar a un consumo de 40 g/día.

\section{Animales, tratamientos y manejo experimental}

Se registró individualmente el peso vivo (PV) al día 100 de gestación de las ovejas diagnosticadas preñadas. Adicionalmente, la condición corporal (CC) de estas ovejas se evaluó por dos personas experimentadas, usando una escala de 1 (muy flacas) al 5 (muy gordas) ${ }^{(15)}$. Dicha información se utilizó para seleccionar 30 ovejas ( $P V=50.8 \pm 0.38$ $\mathrm{kg}$ y $\mathrm{CC}=3.02 \pm 0.04$ unidades), las cuales se asignaron a tres tratamientos $(n=10)$ bajo un diseño de bloques completamente al azar, usando el PV como factor de bloqueo. Los tratamientos consistieron en tres dietas isoenergéticas e isoproteicas, formuladas acorde a los requerimientos recomendados para ovejas gestantes con dos fetos en el último tercio de gestación ${ }^{(16)}$ (Cuadro 1), pero con diferentes niveles de inclusión de AS: 0 (testigo), 3 y $6 \% / \mathrm{kg}$ de materia seca (MS). Las dietas experimentales se ofrecieron diariamente del día 100 de gestación hasta el parto en proporción de $50 \%$ en la mañana $(0700$ h) y $50 \%$ en la tarde (1800 h). El acceso al agua fue ad libitum. Durante el periodo experimental, las ovejas se alojaron en tres corrales, uno por tratamiento, los cuales estaban equipados con comederos, bebederos y sombra. El espacio en comedero fue suficiente para que todas las ovejas pudieran consumir el alimento al mismo tiempo.

El alimento ofrecido y rechazado en cada corral se pesó diariamente para ajustar el consumo de alimento diario cada semana. En la primera semana del experimento se ofrecieron $1.6 \mathrm{~kg}$ de MS/animal, tal como indica el $\mathrm{NRC}^{(16)}$ para ovejas de $50 \mathrm{~kg}$ y que gestan dos fetos. Posteriormente, el consumo de alimento se ajustó para un rechazo promedio diario del $5 \%$ en cada corral. En general, durante el último
Cuadro 1. Ingredientes y composición química de las dietas usadas en la alimentación de las ovejas

\begin{tabular}{lccc}
\hline & \multicolumn{3}{c}{ Nivel de aceite de soya (\%) } \\
\cline { 2 - 4 } Ingredientes de la dieta (\% MS) & 0 & 3 & 6 \\
\hline Aceite de soya & 0.00 & 3.00 & 6.00 \\
Grano de trigo molido & 42.50 & 30.00 & 20.00 \\
Harina de soya & 4.50 & 6.50 & 10.00 \\
Alfalfa henificada molida & 16.00 & 20.00 & 17.00 \\
Paja de trigo & 30.50 & 34.50 & 43.00 \\
Premezcla de vitaminas y minerales & 0.50 & 0.50 & 0.50 \\
Melaza & 4.50 & 4.00 & 2.00 \\
Piedra caliza & 0.60 & 0.60 & 0.60 \\
Ortofosfato & 0.60 & 0.60 & 0.60 \\
Sal común & 0.30 & 0.30 & 0.30 \\
Composición química de la dieta (base seca) & & \\
Materia seca, \% & 92.87 & 93.65 & 93.38 \\
Materia orgánica, \% & 86.48 & 85.20 & 85.38 \\
Proteína cruda, \% & 12.82 & 12.19 & 13.08 \\
Fibra detergente neutro & 43.54 & 47.07 & 49.07 \\
Fibra detergente ácido & 23.95 & 30.54 & 28.25 \\
Cenizas & 9.92 & 11.57 & 9.73 \\
Energía metabolizable, Mcal/kg & 2.42 & 2.44 & 2.46 \\
\hline 1 Porcentaje ofrecido por kg de materia seca. & & & \\
& & &
\end{tabular}

tercio de gestación, las ovejas tratadas con 0,3 y $6 \%$ de AS consumieron $1.35 \pm 0.06,1.33 \pm 0.07 \mathrm{y}$ $1.32 \pm 0.07 \mathrm{~kg}$ de MS/día/animal, respectivamente, asimismo 0, 39.9 y 79.2 g/día/animal de AS, respectivamente.

Muestras de $200 \mathrm{~g}$ de cada dieta se colectaron en bolsas de papel para ser secadas en estufa a 60 - $\mathrm{C}$ por $48 \mathrm{~h}$ cada vez que se prepararon dietas. Al final del periodo experimental, todas las muestras secas se mezclaron en una sola dentro de cada tratamiento, y una sub-muestra de $250 \mathrm{~g}$ de cada dieta se tomó para su posterior análisis bromatológico en el Laboratorio de Nutrición Animal.

\section{Mediciones al parto}

Las ovejas estuvieron en observación continua desde el día 144 de gestación para registrar la información del parto en forma oportuna. Al parto, las crías se identificaron y se registró 
individualmente la siguiente información: sexo, tipo de parto y peso al nacimiento. En la madre se registró individualmente $\mathrm{PV}$ y CC; asimismo en el calostro su peso, volumen, densidad y composición (grasa, proteína y sólidos no grasos [SNG]). Las mediciones y colectas de calostro se realizaron después de que la madre limpió a la cría y antes de que la cría consumiera calostro.

El calostro se colectó totalmente por ordeño manual del medio derecho de la ubre en un vaso de precipitado y aplicando intramuscularmente $10 \mathrm{UI}$ de oxitocina 2 min previo al ordeño(17). El volumen y peso del calostro ordeñado se registraron después de ser multiplicados por 2, considerando el número de tetas. Finalmente, se tomaron muestras de calostro $(40 \mathrm{ml})$ para determinar densidad y porcentaje de proteína, grasa y SNG usando un analizador portátil de leche (Lactichek ${ }^{\mathrm{TM}}$ Rapid Read Ultrasonic Milk Analyzer).

Las variables de productividad de la oveja también se midieron al parto. El tamaño de camada se determinó contando el número de crías nacidas por oveja parida, mientras que el peso de camada se obtuvo sumando los pesos al nacimiento de las crías por oveja parida.

\section{Mediciones postparto}

Se registró individualmente el peso, la ganancia diaria de peso (GDP) y la tasa de mortalidad de los corderos a los 10 días posparto. La GDP se calculó de la siguiente manera: (peso al día 10 - peso al nacimiento)/días transcurridos entre pesajes. La tasa de mortalidad se obtuvo registrando diariamente los corderos muertos en este periodo posparto, y expresando en porcentaje el número de corderos muertos del total de nacidos.

\section{Análisis estadístico}

Con excepción del peso al nacimiento de las crías, todas las variables medidas al momento del parto se sometieron a un análisis de varianza bajo un diseño de bloques completamente al azar. Un análisis de varianza también se aplicó al peso al nacimiento, peso al día 10 posparto y GDP de las crías, pero usando un diseño completamente al azar donde el tamaño de camada fue incluido como covariable. Adicionalmente, las medias se analizaron con polinomios ortogonales para evaluar efectos lineales o cuadráticos conforme el nivel de AS aumentaba. Cuando en el análisis de polinomios ortogonales no se detectaron ( $P>0.05$ ) cambios lineales o cuadráticos significativos en las variables de estudio, las medias se compararon por medio de contrastes ortogonales a una $P<0.05$ : $\mathrm{C} 1$ ) testigo vs inclusión de AS $(3+6 \%)$ y C2) 3 vs $6 \%$ de inclusión de AS. La tasa de mortalidad se analizó con una prueba de J i-cuadrada a una $P<0.05$. En general, la información se analizó usando los procedimientos PROC GLM (análisis de varianza) y PROC FREQ (J icuadrada) del paquete estadístico SAS ${ }^{(18)}$.

Cuadro 2. Peso vivo y condición corporal al parto por efecto del nivel de aceite de soya incluido en la dieta preparto de ovejas de pelo

\begin{tabular}{|c|c|c|c|c|c|c|c|c|}
\hline & \multicolumn{3}{|c|}{ Aceite de soya $(\%)^{1}$} & \multirow{2}{*}{ SEM } & \multicolumn{2}{|c|}{ Efectos $^{2}$} & \multicolumn{2}{|c|}{ Contrastes $^{3}$} \\
\hline & 0 & 3 & 6 & & $\mathrm{~L}$ & C & $\mathrm{C} 1$ & $\mathrm{C} 2$ \\
\hline \multicolumn{9}{|c|}{ Peso vivo (kg): } \\
\hline Inicio & 50.67 & 50.91 & 50.83 & 0.38 & 0.75 & 0.74 & 0.66 & 0.89 \\
\hline Al parto & 49.67 & 49.42 & 50.34 & 1.22 & 0.65 & 0.74 & 0.83 & 0.62 \\
\hline \multicolumn{9}{|c|}{ Condición corporal (1-5): } \\
\hline Inicio & 3.02 & 3.06 & 2.99 & 0.04 & 0.39 & 0.23 & 0.94 & 0.16 \\
\hline Al parto & 2.97 & 3.05 & 3.03 & 0.07 & 0.60 & 0.34 & 0.45 & 0.54 \\
\hline
\end{tabular}




\section{RESULTADOS}

\section{Peso vivo y condición corporal de la oveja}

El PV y la CC al parto no cambiaron ( $P>0.05)$ conforme el nivel de AS incrementó de 0 a $6 \%$ en la dieta preparto (Cuadro 2). Además, el PV y la CC fueron similares ( $P>0.05)$ entre ovejas testigo y alimentadas con AS, así como también entre ovejas alimentadas con 3 y $6 \%$ de AS.

\section{Producción y composición del calostro}

El peso y volumen del calostro incrementaron linealmente $(P<0.05$; Figura 1$)$ mientras que la densidad y los componentes del calostro no cambiaron ( $P>0.05$; Cuadro 3) conforme el nivel de AS aumentó de 0 a $6 \%$ en la dieta preparto. En general, el calostro de ovejas testigo y alimentadas con AS fue similar $(P>0.05)$ en densidad $y$ porcentaje de grasa, proteína y SNG; los mismos resultados se observaron para densidad y composición del calostro ( $P>0.05)$ cuando se compararon ovejas alimentadas con 3 y $6 \%$ de AS.

\section{Productividad de la oveja}

El tamaño y peso de camada por oveja parida no cambiaron ( $P>0.05)$ con el incremento en el nivel

Figura 1. Efecto lineal del peso y volumen del calostro a través de los niveles de inclusión de aceite de soya en el preparto de ovejas de pelo

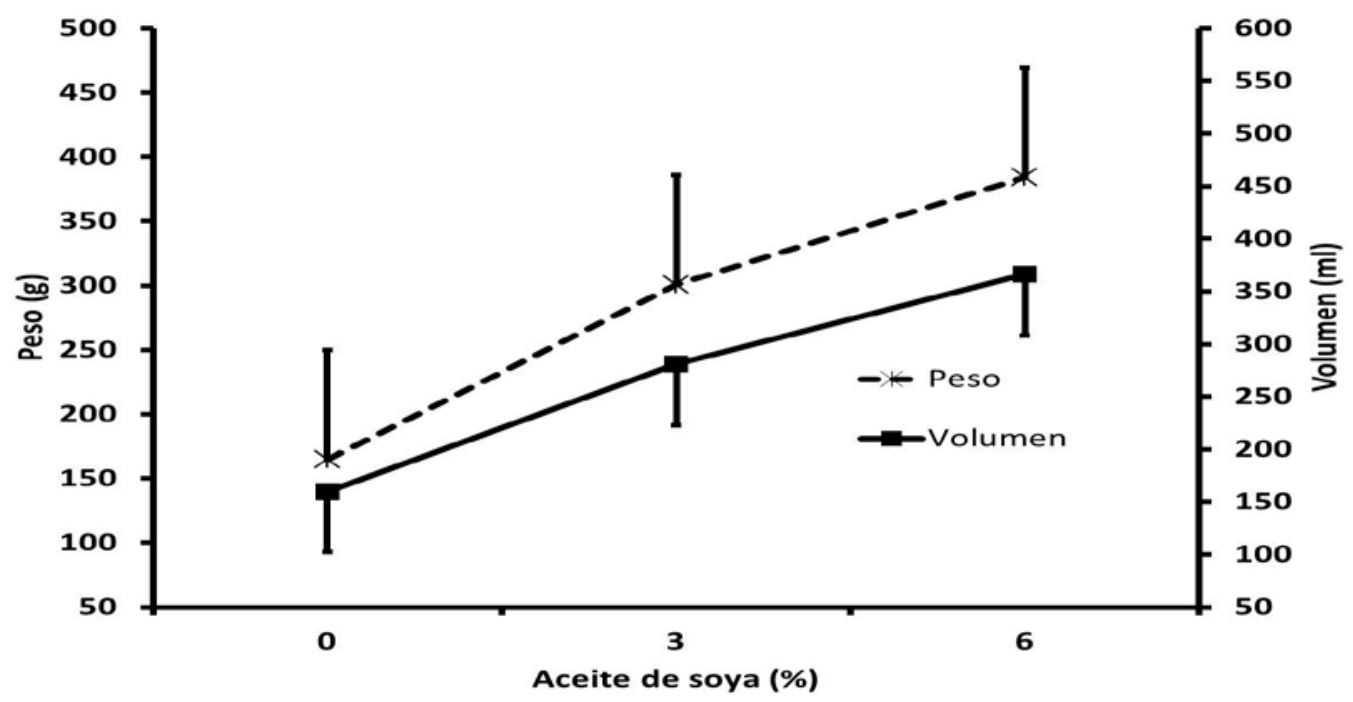

Cuadro 3. Composición del calostro al parto por efecto del nivel de aceite de soya incluido en la dieta pre-parto de ovejas de pelo

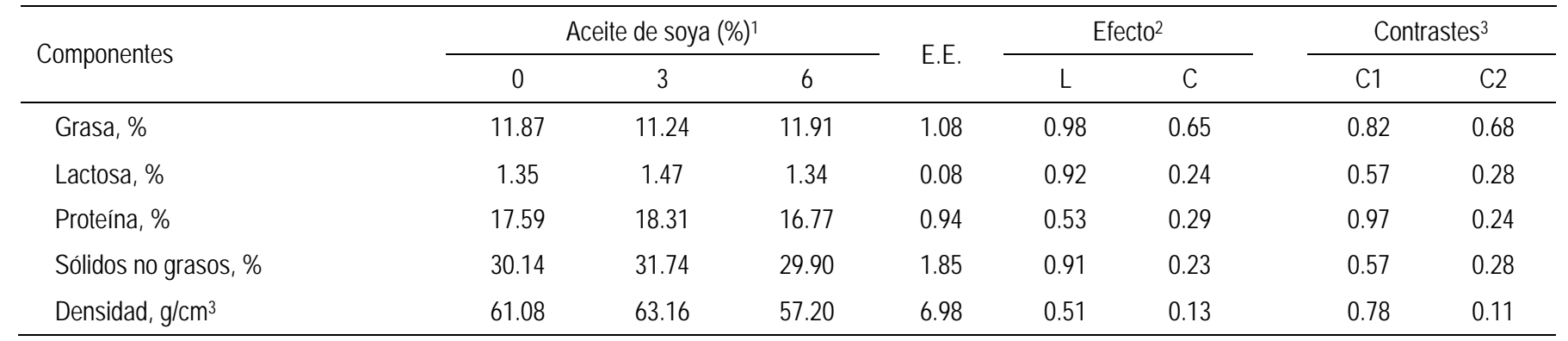

${ }_{1}^{1}$ Porcentaje ofrecido por kilogramo de materia seca.

2 Valores de $P$ debidos a efectos lineales $(L)$ y cuadráticos (C).

${ }^{3}$ Valores de $P$ en los contrastes $C 1$ : testigo vs $3+6 \%$ de aceite de soya; $C 2: 3$ vs $6 \%$ de aceite de soya. 
de AS de 0 a $6 \%$ en la dieta preparto (Cuadro 4). En general, las ovejas testigo comparadas con ovejas alimentadas con AS tuvieron similar ( $P>0.05$ ) tamaño y peso de camada. Las medias de estas variables de productividad de la oveja también fueron similares ( $P>0.05)$ cuando las dietas contenían 3 o $6 \%$ de AS.

\section{Peso al nacimiento y desarrollo de las crías}

El incremento en el nivel de AS en la dieta preparto (0 a $6 \%$ ) no afectó ( $P>0.05$ ) el peso al nacimiento, el peso al día 10 postparto ni la GDP (primeros 10 días postparto) en las crías (Cuadro 4). Estas variables de estudio tampoco se afectaron ( $P>0.05$ ) por incluir AS (testigo vs adición de AS) o por el nivel de inclusión de AS (3 vs $6 \%$ ) en dicha dieta. La tasa de mortalidad de corderos en los primeros 10 días postparto fue similar $(P>0.05)$ entre tratamientos.

\section{DISCUSIÓN}

\section{Peso vivo y condición corporal de la oveja}

En rumiantes alimentados con niveles excesivos de aceites vegetales se puede presentar una reducción en la actividad microbiana ruminal, reflejándose negativamente sobre el consumo de alimento, digestibilidad de la dieta y condición corporal del animal(11). En consecuencia, se recomienda adicionar aceites vegetales en las dietas de ovejas o cualquier rumiante a una dosis óptima para evitar problemas ruminales y pérdidas de peso y condición corporal ${ }^{(14)}$. En este estudio se encontró que las ovejas de genotipo de pelo pueden ser alimentadas hasta con $6 \%$ de AS durante el último tercio de gestación sin afectar su estado corporal al parto, lo cual sugiere que ese nivel de aceite vegetal no altera negativamente la actividad de los microorganismos ruminales $y$, por ende, la ganancia de peso preparto de las ovejas. Cabe mencionar que no se encontraron estudios donde se haya evaluado el desempeño pre- y postparto de ovejas de pelo o lana por efecto de la inclusión de AS u otro aceite vegetal en las dietas pre-parto formuladas isoenergéticas e isoprotéicas. Sin embargo, congruente con los resultados del estudio, el PV y la CC al parto de ovejas de lana no varió cuando se alimentaron durante el preparto con una dieta que contenía $4 \%$ de aceite de pescado ${ }^{(5,6)}$. Al igual que los aceites vegetales, el aceite de pescado es rico en ácidos grasos polinsaturados(14), por lo cual un

Cuadro 4. Desarrollo de las crías y productividad al parto por efecto del nivel de aceite de soya incluido en la dieta pre-parto de ovejas de pelo

\begin{tabular}{|c|c|c|c|c|c|c|c|c|}
\hline & \multicolumn{3}{|c|}{ Aceite de soya $(\%)^{1}$} & \multirow{2}{*}{$\begin{array}{c}\text { Error } \\
\text { Estándar }\end{array}$} & \multicolumn{2}{|c|}{ Efectos $^{2}$} & \multicolumn{2}{|c|}{ Contrastes $^{3}$} \\
\hline & 0 & 3 & 6 & & L & $\mathrm{C}$ & $\mathrm{C} 1$ & $\mathrm{C} 2$ \\
\hline \multicolumn{9}{|l|}{ Productividad al parto: } \\
\hline Tamaño de camada, $n$ & 1.50 & 1.74 & 1.54 & 0.19 & 0.88 & 0.39 & 0.54 & 0.49 \\
\hline Peso de camada, kg & 4.70 & 5.88 & 5.59 & 0.45 & 0.16 & 0.22 & 0.07 & 0.67 \\
\hline \multicolumn{9}{|l|}{ Desarrollo de corderos: } \\
\hline Número de crías, n & 16 & 14 & 14 & --- & --- & --- & --- & --- \\
\hline Peso al nacimiento, kg & 3.14 & 3.33 & 3.52 & 0.13 & 0.41 & 0.76 & 0.39 & 0.87 \\
\hline Peso a 10 d postparto, kg & 6.27 & 6.27 & 5.95 & 0,27 & 0.24 & 0.70 & 0.41 & 0.36 \\
\hline Ganancia diaria de peso, g & 299 & 284 & 243 & 22 & 0.16 & 0.85 & 0.20 & 058 \\
\hline Tasa de mortalidad, $\%^{4}$ & 6.25 & 0.00 & 0.00 & --- & --- & --- & --- & --- \\
\hline
\end{tabular}

1 Porcentaje ofrecido por $\mathrm{kg}$ de materia seca

2 Valores de $P$ debidos a efectos lineales (L) y cuadráticos (C).

3 Valores de $P$ en los contrastes $C 1$ : testigo vs $3+6 \%$ de aceite de soya; $C 2: 3$ vs $6 \%$ de aceite de soya.

${ }^{4}$ La tasa de mortalidad no fue afectada por tratamiento $(P>0.05)$. 
efecto similar de estos aceites podría esperarse sobre el estado corporal al parto cuando son adicionados a la dieta de ovejas gestantes en el último tercio.

\section{Producción y composición del calostro}

En general, la inclusión de AS en la dieta preparto resultó benéfica para mejorar la producción de calostro al parto, siendo mayor tanto el peso como el volumen de calostro cuando las ovejas consumieron la dieta con $6 \%$ de AS. Poco se ha estudiado al respecto, no obstante, en un estudio previo $^{(19)}$ se indicó que la producción de calostro al parto no fue afectada en ovejas multíparas Katahdin x Pelibuey por adicionar $6.5 \%$ de AS a la dieta ofrecida durante los últimos 50 días de gestación. También existen reportes sobre el incremento en la producción de leche por incluir en la dieta de ovejas $^{(20,21)}$ y cabras ${ }^{(22)}$ lactantes un 3 y $5 \%$ de AS, respectivamente. Por lo tanto, considerando que $6.5 \%$ de AS en la dieta preparto no altera la producción de calostro en las ovejas de pelo(19), los resultados de este trabajo muestran que la inclusión de $6 \%$ de AS en la dieta ofrecida en el último tercio de gestación es adecuada para mejorar la producción de calostro en ovejas Katahdin $\mathrm{x}$ Pelibuey.

Es importante mencionar que no existe una explicación precisa en relación al aumento en la producción de calostro al parto conforme la dosis de AS incrementó de 0 a $6 \%$ en la dieta preparto. Algunos estudios ${ }^{(21,23)}$ señalan que la suplementación de aceites vegetales mejora la producción de leche en ovejas y cabras debido a que aumenta el nivel de energía en la dieta. También se ha reportado que la inclusión de aceites vegetales en dietas formuladas isoenergética e isoprotéicamente provoca que se incluya un mayor nivel de forraje $\mathrm{e}^{(24,25)}$. Lo anterior puede promover una interacción positiva entre el aceite vegetal adicionado y la mayor cantidad de forraje de las dietas para incrementar la producción de ácidos grasos volátiles, principalmente de acetato y butírico, los cuales se relacionan con una mejor secreción láctea en ovejas ${ }^{(14,26)}$. Por lo tanto, considerando que en este estudio las dietas contenían similar nivel de energía y proteína, probablemente la mayor producción de calostro con el nivel más alto de AS se debió a un efecto favorable de la interacción entre el nivel de AS y la cantidad de forraje en la dieta, pero no a un efecto directo del AS. Esto considerando que la dieta de $6 \%$ de AS se formuló con 5.5 y $13.5 \%$ más forraje que las dietas de 0 y $3 \%$ de AS, respectivamente. Cabe aclarar que no se encontró algún reporte previo que indicara esta relación entre nivel de forraje y AS en la dieta de preparto para mejorar la producción de calostro en ovejas.

La composición y la densidad del calostro no se alteraron por incluir 3 o $6 \%$ de AS en la dieta preparto de ovejas de pelo. Estos resultados coinciden con lo observado en otro estudio ${ }^{(3)}$ donde la inclusión de $5 \%$ de aceite de girasol en la dieta preparto de ovejas Rahmani no afectó el contenido de energía en calostro, ni los porcentajes de proteína, grasa ni SNG en el mismo. Sin embargo, Reyes ${ }^{(19)}$ reportó que el calostro de ovejas de pelo suplementadas con AS (6.5\%) en el preparto tuvo similar densidad y contenido de proteína y SNG, pero menor contenido de grasa comparado con el calostro de ovejas testigo (sin AS). El autor de ese estudio(19) indicó que la caída en el porcentaje de grasa del calostro por efecto del AS fue un indicativo de que el nivel de aceite usado en la dieta preparto fue alto, lo cual explica la discrepancia con el resultado de porcentaje de grasa del presente estudio. La inclusión dietaria de elevada cantidad de AS provoca que incremente la concentración del ácido graso C18:2 trans-10 cis-12 a nivel de biohidrogenación ruminal, el cual inhibe la enzima desaturasa- $\Delta 9$ en la glándula mamaria y estimula una reducción en la síntesis de ácidos grasos de cadena corta y mediana, reflejándose en una caída en el contenido de grasa láctea(13). Cabe mencionar que estos compuestos intermedios de la biohidrogenación del AS solamente influyen en el contenido de grasa, pero no en otros componentes del calostro, acorde a lo indicado por otros autores ${ }^{(27)}$.

\section{Productividad de la oveja}

El peso de la camada al parto no varió con el incremento en el nivel de inclusión de AS en la dieta 
preparto. Este resultado era esperado considerando que el peso al nacimiento de las crías y el tamaño de camada al parto no se afectaron por el nivel de AS en la dieta de las ovejas durante el preparto. En general, los promedios de peso y tamaño de camada encontrados en este estudio fueron de $5.4 \mathrm{~kg}$ y 1.6 crías/oveja parida, respectivamente. Estos promedios de peso y tamaño de camada al parto coinciden con lo encontrado en estudios previos $^{(2,28,29)}$ realizados en ovejas de pelo bajo las mismas condiciones climáticas y de producción.

\section{Peso al nacimiento y desarrollo de las crías}

El AS es rico en ácidos grasos polinsaturados $\mathrm{n}-6$, específicamente el linoleico ${ }^{(30)}$. Estos ácidos grasos son metabolizados dentro del cuerpo del animal en ácido araquidónico, el cual es precursor de la síntesis de prostaglandinas $E_{2}$ y $F_{2 a}$ en el tejido uterino y placentario(10). Así, en ovejas alimentadas en el preparto con dietas altas en $A S$, se ha observado la presencia de partos prematuros producto de un incremento en niveles circulantes sanguíneos de prostaglandinas ${ }^{(9,31,32)}$. En ovinos, el parto prematuro se considera como una de las principales causas de morbilidad, mortalidad y bajo crecimiento de las crías en los primeros días posparto ${ }^{(33)}$. En este estudio, no se observó efecto alguno por la adición de 3 o $6 \%$ de AS a la dieta preparto sobre el peso al nacimiento, la tasa de mortalidad ni en el crecimiento de los corderos en los primeros días postparto, lo cual coincide con lo reportado en ovejas Katahdin $x$ Pelibuey ${ }^{(2)}$ y cerdas $^{(34)}$, cuando fueron alimentadas durante las últimas semanas antes del parto con una dieta que contenía 6.5 y $3 \%$ de AS, respectivamente. Por lo tanto, los resultados de esta investigación muestran que ovejas de pelo en el último tercio de su gestación se pueden alimentar hasta con $6 \%$ de AS; esto sin tener problemas de partos prematuros que afecten negativamente la tasa de sobrevivencia, el peso al nacimiento o el crecimiento de las crías en su vida postnatal.

Cabe mencionar que la tasa de mortalidad de corderos a los 10 días posparto no se afectó por la inclusión de AS en la dieta preparto, lo cual puede deberse al bajo número de corderos usados por tratamiento para hacer el análisis. Sin embargo, se destaca que ningún cordero murió cuando las ovejas se alimentaron con las dietas que contenían 3 o $6 \%$ de AS, mientras que en ovejas testigo se observó $6.5 \%$ de mortalidad en corderos durante los primeros 10 días posparto.

\section{CONCLUSIONES E IMPLICACIONES}

El aceite de soya a niveles de 3 o $6 \%$ puede usarse como una fuente de energía en dietas de preparto para ovejas de pelo, sin que esto implique un efecto negativo sobre el peso de las ovejas o la composición del calostro al parto, ni en el desarrollo de las crías en el periparto. Se recomienda formular las dietas de preparto con $6 \%$, ya que a este nivel se obtiene mayor producción de calostro, lo cual puede resultar benéfico para mejorar el sistema inmunológico de las crías y garantizar la sobrevivencia de las mismas.

\section{AGRADECI MI ENTOS}

Se agradece a la UABC por el apoyo otorgado para el desarrollo de esta investigación dentro del marco de la 17va. Convocatoria Interna de Apoyo a Proyectos de Investigación. También, los primeros tres autores agradecen al Conacyt, México, por el financiamiento otorgado para la realización de sus estudios de posgrado en el ICA-UABC.

\section{LITERATURA CITADA}

1. Banchero GE, Quintans G, Martin GB, Milton JTB, Lindsay DR. Nutrition and colostrum production in sheep. 2. Metabolic and hormonal responses to different energy sources in the final stage of pregnancy. Reprod Fert Develop 2004; 16(6):645-653.

2. Vicente-Pérez R, Avendaño-Reyes L, Álvarez FD, Correa-Calderón A, Meza-Herrera CA, Mellado M, Quintero JA, et al. Comportamiento productivo, consumo de nutrientes y productividad al parto de ovejas de pelo suplementadas con energía en el preparto durante verano e invierno. Arch Med Vet 2015;47(3):301-309.

3. Abd-Allah M. Effects of parity and nutrition plane during late pregnancy on metabolic responses, colostrum production and lamb output of Rahmani ewes. Egyptian J Anim Prod 2013;50(3):132142.

4. De Pablo MA, De Cienfuegos G. Modulatory effects of dietary lipids on immune system functions. Immunol Cell Biol 2000; 78(1):31-39. 
5. Annett RW, Dawson LER, Edgar H, Carson AF. Effects of source and level of fish oil supplementation in late pregnancy on feed intake, colostrum production and lamb output of ewes. Anim Feed Sci Tech 2009; 154(3-4):169-182.

6. Capper J L, Wilkinson RG, Mackenzie AM, Sinclair LA. Polyunsatured fatty acid supplementation during pregnancy alters neonatal behavior in sheep. J Nutr 2006; 136(2):397-403.

7. Andrews J, Vazquez-Añón $M$, Bowman $G$. Fat stability and preservation of fatty acids with AGRADO antioxidant in feed ingredients used in ruminant rations. J Dairy Sci 2006;89(Suppl 1):60.

8. Acuti G, Trabalza-Marinucci M, Cagiola M, Pela M, Curina G, Moscati L. Extruded linseed supplementation in the diet of dairy sheep: The effects on inmmune response and oxidative stress as affected by the physiological state. Small Ruminant Res 2012;106S:S21-S28.

9. Elmes M, Green LR, Poorce K, Newman J, Burrage D, Abayasekara $\mathrm{DRE}$, et al. Raised dietary n-6 polyunsaturated fatty acid intake increases 2-series prostaglandin production during labour in the ewes. J Physiol 2005; 562(Pt2):583-592.

10. Wathes DC, Abayasekara DRE, Aitken RJ. Polyunsaurated fatty acids in male and female reproduction. Biol Reprod 2007; 77(2):190-201.

11. J enkins TC, Wallace RJ, Moate PJ, Mosley EE. Board-invited Review: Recent advances in biohydrogenation of unsaturated fatty acids within the rumen microbial ecosystem. J Anim Sci 2008;86(2):397412.

12. Shingfield $\mathrm{KJ}$, Bernard L, Leroux $\mathrm{C}$, Chilliard $\mathrm{Y}$. Role of trans fatty acids in the nutritional regulation of mammary lipogenesis in ruminants. Animal 2010;4(7):1140-1166.

13. Almeida OC, Pires AV, Susin I, Gentil RS, Méndez CQ, Queiroz MAA, et al. Milk fatty acids profile and arterial blood milk fat precursors concentration of dairy goats fed increasing doses of soybean oil. Small Ruminant Res 2013;114(1);152-160.

14. Bauman DE, Baumgard LH, Corl BA, Griinari JM. Biosynthesis of conjugated linoleic acid in ruminants. J Anim Sci 2000;77(ESuppl):1-15.

15. Russel AJ F, Doney J M, Gunn RJ. Subjective assessment of body fat in live sheep. J Agr Sci 1969; 72(3):451-454.

16. NRC. Nutrient requirements of small ruminants: Sheep, goats, cervids, and new world camelids. National Academy of Science, Washington, DC; 2007.

17. Bencini R, Hatmann PE, Lighfoot RJ . Comparative dairy potential of Awassi $x$ Merino and Merino ewes. Proc Australian Assoc Anim Breed Genetic 1992;10:114-117.

18. SAS INSTITUTE, SAS/STAT. User's guide statistics released 9.1, 2nd Ed. SAS Institute, Inc. Cary, NC, USA; 2004.

19. Reyes MF. Suplementación energética preparto en ovejas de pelo estresadas por calor: Efecto sobre galactogénesis y crecimiento de las crías [tesis maestría]. Instituto de Ciencias Agrícolas, UABC; 2014.

20. Manso T, Bodas R, Vieira C, Mantecón AR, Castro T. Feeding vegetable oils to lactanting ewes modifies the fatty acid profile of suckling lambs. Animal 2011;5(10):1659-1667.

21. Titi HH, Al-Fataftah AR. Effect of supplementation with vegetable oil on performance of lactating Awassi ewes, growth of their lambs and on fatty acid profile of milk and blood of lambs. Archv Tierzucht 2013;56(45):467-479.

22. Seong-Ho C, J ong-Kyu K, Hong-Gu L, Chang-Weon C, Yang-II C, Man-Kang S. Cis-9, trans-11- conjugated linoleic acid in dairy goat milk was increased by high linoleic (soybean oil) or linolenic (linseed oil) acid diet. Korean J Food Sci An 2013;33(4):487-492.

23. Martínez MAL, Núñez SN, Garzón SAl, Peña BF, Domenech GV, Hernández RF. Metaanálisis del uso de semillas y aceites en la dieta de ovejas y cabras. Pesq Agropec Bras 2015;50(9):821-828.

24. Martínez MAL, Pérez-Hernández M, Pérez-Alba LM, Carrión-Pardo D, Gómez-Castro AG. Adición de aceites vegetales a la dieta de cabras lecheras: Efecto sobre la digestibilidad y los resultados productivos. Arch Med Vet 2012; 44(1):21-28.

25. Vargas-Bello-Pérez E, Vera RR, Aguilar C, Lira R, Peña I, Valenzuela A, Cerda H. Effect of dietary inclusion of lampante olive oil on milk and cheese fatty acid profiles of ewes. Int J Fats Oils 2013;64(3):295-303.

26. Mele M, Buccioni A, Petacchi F, Serra A, Banni S, Antongiovanni M, Secchiari P. Effect of forage/concentrate ration and soybean oil supplementation on milk yield and composition from Sarda ewes. Anim Res 2006; 55:273-285.

27. Li XZ, Yan CG, Long RJ, J in GL, Shine Khuu J, J i BJ , et al. Conjugated linoleic acid in rumen fluid and milk fat, and methane emission of lactating goats fed a soybean oil-based diet supplemented with sodium bicarbonate and monensin. Asian-Aust J Anim Sci 2009;22(11): 1521-1530.

28. Macías-Cruz U, Álvarez-Valenzuela FD, Correa-Calderón A, MolinaRamírez L, González-Reyna A, Soto-Navarro SA, Avendaño-Reyes L. Pelibuey ewe productivity and subsequent pre-weaning lamb performance using hair-sheep breed under a confinement system. J Appl Anim Res 2009;36(2):255-260.

29. Macías-Cruz U, Álvarez-Valenzuela FD, Olguín-Arredondo HA Molina-Ramírez L, Avendaño-Reyes L. Ovejas Pelibuey sincronizadas con progestágenos y apareadas con machos de razas Dorper y Katahdin bajo condiciones estabuladas: producción de la oveja y crecimiento de los corderos durante el período predestete. Arch Med Vet 2012;44(1):29-37.

30. Jain VP, Proctor A, LallR. Pilot-scale production of conjugated linoleic acid-rich soy oil by photoirradiation. J Food Sci 2008; 73(4):183-192.

31. Challis JRG, Sloboda DM, Alfaidy N, Lye SJ, Gibb W, Patel FA, et al. Prostaglandins and mechanisms of preterm birth. Reproduction 2002; 124(1): 1-17.

32. Cheng Z, Elmes M, Kirkup SE, Chin EC, Abayasekara DRE, Wathes DC. The effect of a diet supplemented with the $n-6$ polyunsaturated fatty acid linoleic acid on prostaglandin production in early- and late-pregnant ewes. J Endocrinol 2005; 184(1):165-178.

33. Challis JRG, Matthews SG, Gibb W, Lye SJ. Endocrine and paracrine regulation of birth at term and preterm. Endocr Rev 2000;21(5):514-550.

34. Rooke JA, Bland IM, Edwards SA. Relationships between fatty acid status of sow plasma and that of umbilical cord, plasma and tissues of newborn piglets when sows were fed on diets containing tuna oil or soybean oil in late pregnancy. British J Nutr 1999;82(3):213-221. 